\title{
Compartment Syndrome Secondary to Knee Lipohemarthrosis
}

\author{
Madeleine E. Kim ${ }^{1}$, Thor S. Stead ${ }^{2}$, Latha Ganti ${ }^{3,4,5,6}$ \\ 1. Emergency Medicine, Baylor School, Chattanooga, USA 2. Epidemiology and Public Health, Alpert Medical School of \\ Brown University, Providence, USA 3. Emergency Medicine, Envision Physician Services, Plantation, USA 4. Emergency \\ Medicine, University of Central Florida College of Medicine, Orlando, USA 5. Emergency Medicine, Ocala Regional \\ Medical Center, Ocala, USA 6. Emergency Medicine, HCA Healthcare Graduate Medical Education Consortium \\ Emergency Medicine Residency Program of Greater Orlando, Orlando, USA
}

Corresponding author: Latha Ganti, latha.ganti@ucf.edu

\begin{abstract}
When treating patients presenting with knee trauma or intra-articular fracture, clinicians should maintain a high index of suspicion for lipohemarthrosis. Diagnosis of lipohemarthrosis can be accomplished via visualization of a fat-fluid level. Increased fluid and pressure build-up within the joint space may lead to compartment syndrome, which requires emergency compartment fasciotomy. In this paper, we discuss the importance of identifying lipohemarthrosis in patients presenting with intra-articular fracture, as well as the necessity of frequent patient re-evaluations in order to monitor the onset of compartment syndrome.
\end{abstract}

Categories: Emergency Medicine, Orthopedics, Trauma

Keywords: lipohemarthrosis, tibial fracture, compartment syndrome, fat-fluid level, fibular fracture, intra-articular fracture, compartment pressure

\section{Introduction}

Knee traumas account for over 500,000 visits to the emergency department (ED) per year in the United States [1-3]. When displaced, intra-articular fractures may result in bone marrow and blood spilling into the surrounding joint capsule [4]. This is defined as lipohemarthrosis. Lipohemarthrosis is not entirely uncommon in intra-articular fractures, occurring in 35\%-41\% of cases $[1,5,6]$. In the knee, lipohemarthrosis is most likely to accompany tibial plateau fractures and may require orthopedic surgery in certain cases [7]. Lipohemarthrosis can be diagnosed with plain radiography (X-rays) or computed tomography (CT) of the injured knee, with axial CT images oftentimes allowing visualization of fat-fluid levels within the joint [1]. When intra-articular fractures are displaced and bone marrow and blood spill into the joint, the differing densities of the bone marrow and blood cause separation of fat and fluid, forming the fat-fluid levels visible in scans. Over time, the fluid can further separate into serum and red blood cell precipitate layers, forming three layers referred to as double fluid-fluid levels [1]. Build-up of fluid within a joint may lead to compartment syndrome, a limb-threatening complication [8]. Thus, it is necessary for clinicians to have a high index of suspicion for lipohemarthrosis and therefore compartment syndrome when treating patients with knee trauma. In this paper, we discuss a case in which a patient presented with an intra-articular tibial

Review began 07/23/2021 Review ended 07/25/2021 Published 08/06/2021

\section{(c) Copyright 2021}

Kim et al. This is an open access article distributed under the terms of the Creative Commons Attribution License CC-BY 4.0., which permits unrestricted use, distribution, and reproduction in any medium, provided the original author and source are credited. fracture and a right proximal fibular fracture, as well as signs and symptoms of early compartment syndrome due to lipohemarthrosis in subsequent re-evaluations.

\section{Case Presentation}

A 30-year-old male presented to the ED via emergency medical services (EMS) with right knee pain and swelling after sustaining a fall. The patient stated that he was standing on a conveyor belt on his left lower extremity and then fell, landing with his right knee in varus stress. The fall happened from approximately 3 feet, and upon falling, he felt an immediate pop, accompanied by intense pain that radiated distally down to his foot. The patient reported the severity of pain at onset as 10/10 and was unable to bear weight on his right lower extremity. En route, EMS reported that the patient received 100 mcg of intravenous (IV) fentanyl, with improvement in pain from $10 / 10$ to $2 / 10$, as well as a 500 cc bolus of IV saline.

The patient had a temperature of $36.5^{\circ} \mathrm{C}$, with a blood pressure of $135 / 85 \mathrm{mmHg}$ and a pulse of 53 beats per minute. Electrocardiogram (ECG) showed slight sinus bradycardia but the patient was asymptomatic. Oxygen saturation was $95 \%$ on room air, and respiratory rate was 14 breaths per minute. Though otherwise healthy, the patient did admit to smoking a pack of cigarettes every day. Physical examination showed no abnormalities outside of the affected extremity. Examination of the right lower extremity showed moderate swelling over the right anterior knee with moderate tenderness to palpation over the right anterior and right lateral knee. There was moderate tenderness to the right proximal fibula, but the anterior compartments were soft, and skin was intact. The patient's calf was not tender. His right foot was diffusely tender to palpation, but otherwise had no swelling, erythema, or ecchymosis. The patient was able to move all digits and his dorsalis pedis and posterior tibial pulses were equal bilaterally. On admission, the patient was agitated but otherwise alert and oriented. 


\section{Cureus}

$\mathrm{X}$-ray of the right femur showed right tibial and fibular fractures without femoral fracture. X-ray of the right foot showed no evidence of acute fracture or dislocation. X-ray of the right tibia fibula showed comminuted proximal tibial and fibular fractures with evidence of intra-articular extension, right knee joint effusion, and lipohemarthrosis (Figure 1).

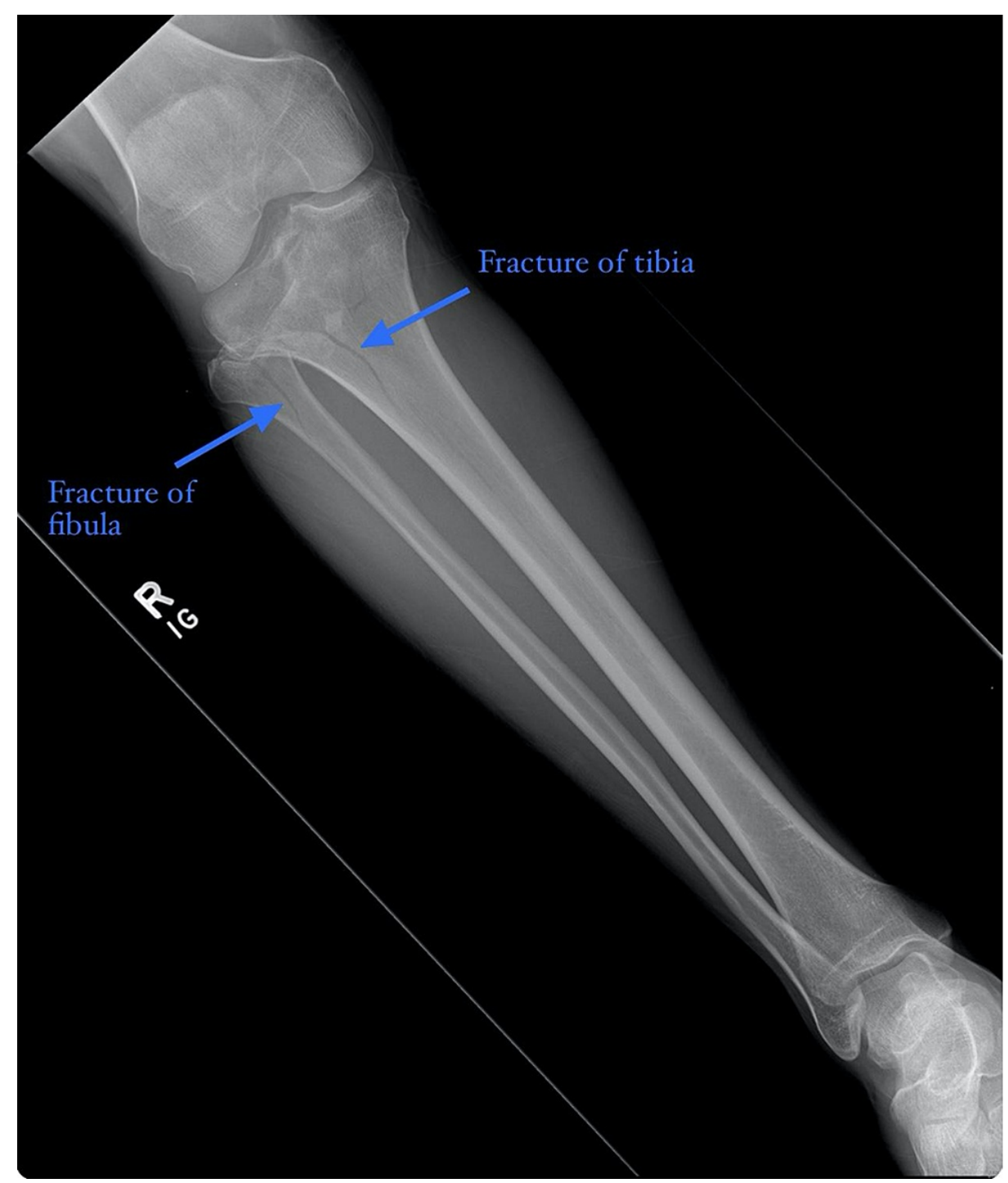

FIGURE 1: Plain radiograph of the right tibia fibula showed comminuted proximal tibial and fibular fractures (blue arrows) with evidence of intraarticular extension, right knee joint effusion, and lipohemarthrosis

A CT of the lower extremity without contrast yielded evidence of a complex comminuted depressed fracture of the proximal tibia, involving mainly the lateral plateau, as well as a fracture of the fibular head. Moderate lipohemarthrosis and soft tissue edema was also identified (Figure 2). 


\section{Cureus}

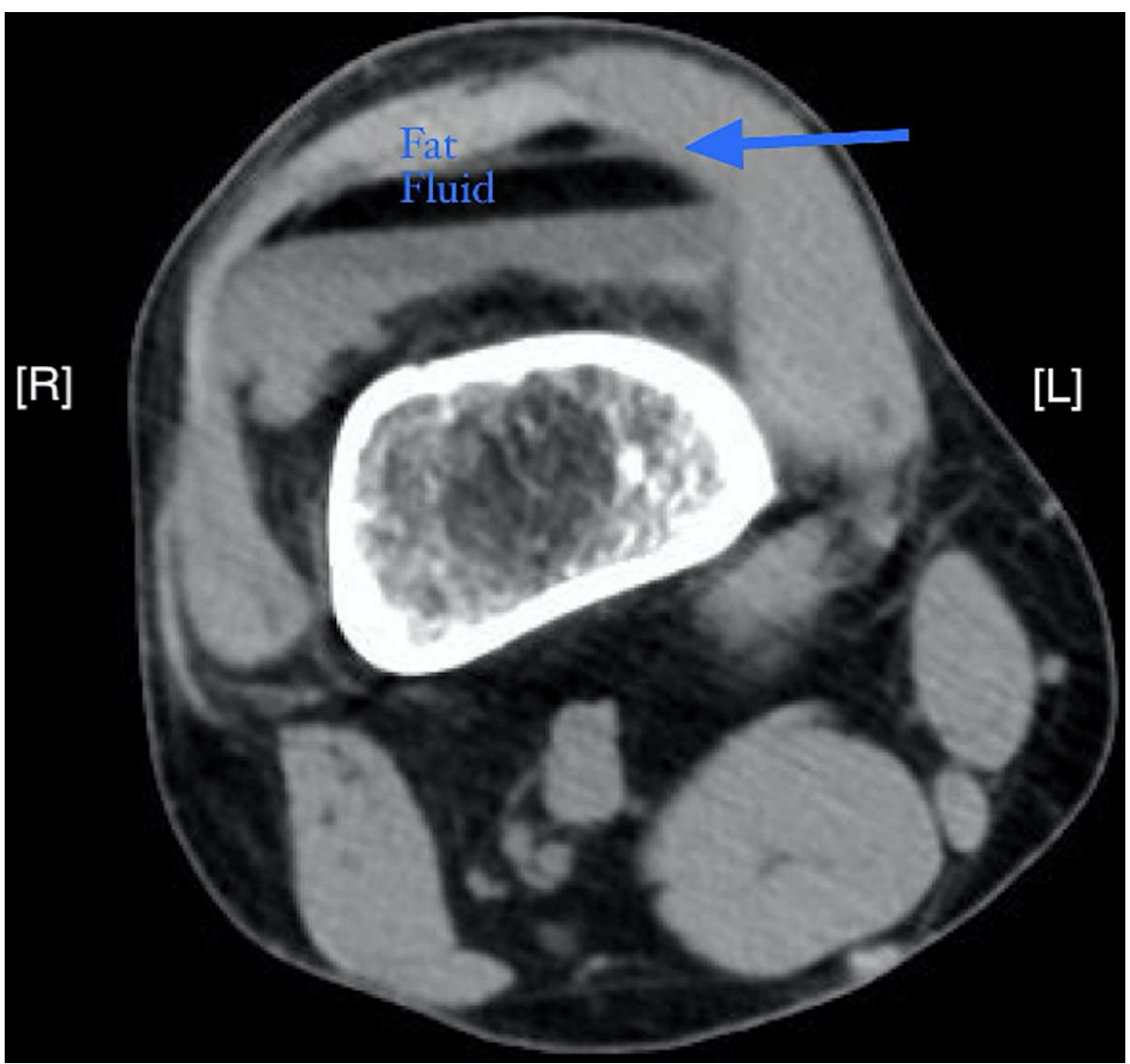

FIGURE 2: Axial CT image without contrast of the right lower extremity showed the fat-fluid level (blue arrow) indicative of lipohemarthrosis.

Along with moderate lipohemarthrosis, soft tissue edema was identified. CT also revealed evidence of complex comminuted depressed fracture of the proximal tibia, involving mainly the lateral plateau. There is also a fracture of the fibular head

The patient was diagnosed with a right proximal intra-articular tibial fracture (tibial plateau fracture), right knee effusion, right proximal fibular fracture, and lipohemarthrosis. Re-evaluations of the patient at first revealed no signs or symptoms of compartment syndrome, but by the fourth re-evaluation, the patient presented with anterior compartments of the right lower extremity warm and firm to touch. The extremity was placed in an increased level of elevation and was iced, and a CT without contrast was ordered following observed signs of early compartment syndrome. At the time of the fourth re-evaluation, the patient's compartment pressure was measured at $62 \mathrm{mmHg}$. ED staff continued to assess the compartment pressures of the patient with a commercially available manometer (Table 1 ). 


\section{Cureus}

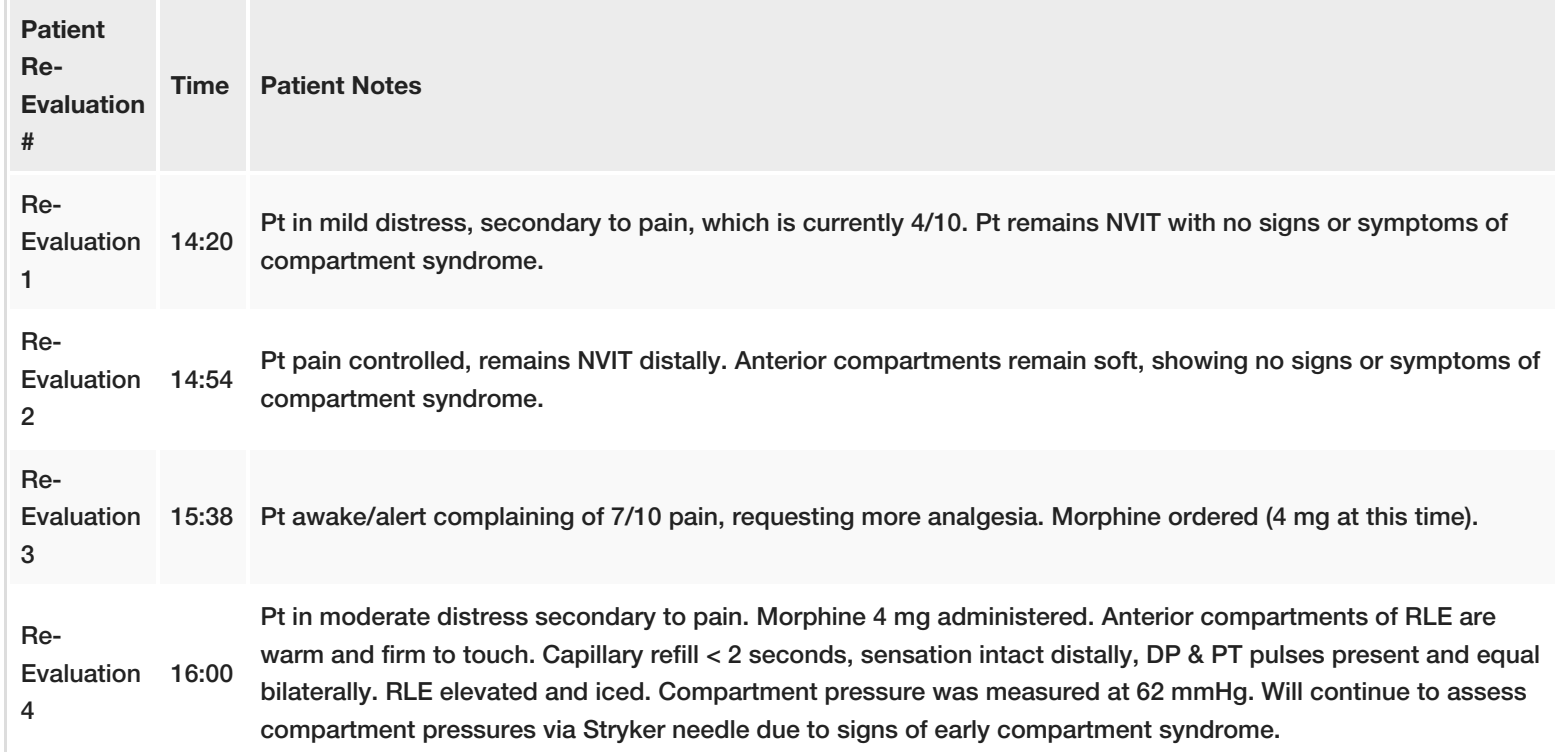

\section{TABLE 1: Table delineating re-evaluations of the patient including time compartment pressure recorded at each re-evaluation}

pt: patient; NVIT: neurovascularly intact; RLE: right lower extremity; DP \& PT: dorsalis pedalis \& posterior tibial

Following a diagnosis of early compartment syndrome in the patient, an emergency right lower extremity four-compartment fasciotomy was planned and the operating room (OR) team was called in. A multi-planar knee-spanning external fixator system was placed for soft tissue and bone stabilization. The patient returned to the OR on day 3 and 4 for repeat irrigation and debridement of the fasciotomies, as well as removal of the external fixator and open reduction internal fixation of the patient's tibial plateau fracture and fasciotomy closure. Antibiotics were continued for 24 hours following the completion of the patient's fasciotomies. The patient remained in the hospital for ten days improved pain control and rehabilitation. He was discharged home with home health care.

\section{Discussion}

It is known that the most common cause of lipohemarthrosis is traumatic knee injury, with lipohemarthrosis even being considered pathognomonic for acute fracture [1]. Considering the fact that knee traumas account for hundreds of thousands of ED visits per year, it is necessary that clinicians maintain a high index of suspicion for lipohemarthrosis when patients present with knee traumas or intra-articular fractures. This urgency is only compounded by the additional risk of compartment syndrome that may accompany lipohemarthrosis. The extrusion of fat and blood from the fracture causes pressure build-up within the joint space, leading to increased compartment pressures within the joint [9]. As pressure increases within the closed fascial space, blood flow, and tissue perfusion reduce, leading to nerve and muscle dysfunction, extremity deformities, paralysis, and in some cases, amputation if the compartment syndrome is left untreated $[10,11]$. Surgical interventions like compartment fasciotomies may also be required. Therefore, due to the grave consequences of untreated compartment syndrome, it is of utmost importance that clinicians are able to recognize lipohemarthrosis and its potential resultant compartment syndrome in patients.

X-rays may allow for the detection of lipohemarthrosis; however, they are less sensitive than other imaging modalities [1]. Because of this, CTs are considered the gold standard for lipohemarthrosis visualization because they allow for easier identification of the tell-tale fat-fluid levels formed when fat and blood from the fracture separate within the joint space [1]. While MRIs are also helpful for the identification of fat-fluid levels, the decreased availability and increased price of this form of imaging make it less desirable, and thus CTs without contrast are considered sufficient when attempting to visualize lipohemarthrosis.

The particular case described in this paper is unique in that the patient sustained a right proximal intraarticular tibial fracture, right knee effusion, right proximal fibular fracture, and developed lipohemarthrosis after falling only 3 feet during a workplace accident. While landing on his right lower extremity in varus stress likely played a part in the severity of his injuries, the patient's daily smoking habits are also notable when exploring the traumatic nature of his injury. Research demonstrates that tobacco smoking has a negative effect on bone turnover and may cause lower bone mass, leading to increased risk of fracture, osteoporosis, and certain skeletal system disorders [12]. 
Another notable aspect of this particular case is its clear delineation of the onset of compartment syndrome. Though the patient did not show signs of compartment syndrome upon admission to the ED, and though the first, second, and third re-evaluations of the patient did not yield any signs or symptoms of compartment syndrome, upon the fourth re-evaluation, his right lower extremity was warm and firm to touch, and his compartment pressure was measured at $62 \mathrm{mmHg}$. The onset of this early compartment syndrome took only 1 hour and 45 minutes following time of admittance to the ED. This case demonstrates that continual neurovascular and pressure checks of patients diagnosed with lipohemarthrosis are necessary, especially since onset of compartment syndrome can occur fairly rapidly and requires urgent intervention (eg. compartment fasciotomy) following diagnosis.

\section{Conclusions}

When treating patients presenting with knee trauma or intra-articular fractures, it is important that clinicians maintain a high index of suspicion for lipohemarthrosis and onset of early compartment syndrome. X-ray and CT imaging allows for visualization of intra-articular fractures, as well as the classic fat-fluid levels that indicate lipohemarthrosis. Frequent re-evaluations of patients diagnosed with lipohemarthrosis are necessary, especially since the resultant fluid and pressure build-up within joint spaces may lead to the development of compartment syndrome in patients. Checking compartment pressures with a manometer allows for the monitoring of compartment syndrome progression in order to ensure that surgical intervention via compartment fasciotomy occurs in a timely manner if indicated.

\section{Additional Information \\ Disclosures}

Human subjects: Consent was obtained or waived by all participants in this study. HCA Centralized Algorithms for Research Rules on IRB Exemptions (CARRIE)/ IRB manager issued approval 2021-526. HCA Centralized Algorithms for Research Rules on IRB Exemptions (CARRIE)/IRB manager issued approval [2021-526]. Based on the information provided and attested as true, the research plan described does not require IRB oversight. This is because you are either a) not engaging in research with human subjects as defined by federal regulations; b) engaging in research with human subjects deemed excluded from IRB oversight per 45CFR46.102(1) OR c) engaging in research with sufficient human subject protections in the design to meet one or more IRB exemption criteria set forth in 45CFR46.104. Conflicts of interest: In compliance with the ICMJE uniform disclosure form, all authors declare the following: Payment/services info: All authors have declared that no financial support was received from any organization for the submitted work. Financial relationships: All authors have declared that they have no financial relationships at present or within the previous three years with any organizations that might have an interest in the submitted work. Other relationships: All authors have declared that there are no other relationships or activities that could appear to have influenced the submitted work.

\section{Acknowledgements}

This research was supported (in whole or in part) by HCA Healthcare and/or an HCA Healthcare affiliated entity. The views expressed in this publication represent those of the author(s) and do not necessarily represent the official views of HCA Healthcare or any of its affiliated entities.

\section{References}

1. Davis DL, Vachhani P: Traumatic extra-capsular and intra-capsular floating fat: fat-fluid levels of the knee revisited. J Clin Imaging Sci. 2015, 5:60. 10.4103/2156-7514.170729

2. Aponte EM, Novik JI: Identification of lipohemarthrosis with point-of-care emergency ultrasonography: case report and brief literature review. J Emerg Med. 2013, 44:453-6. 10.1016/j.jemermed.2012.07.062

3. McCaig LF, Nawar EW: National Hospital Ambulatory Medical Care Survey: 2004 emergency department summary. Adv Data. 2006, 23:1-29.

4. Aklilu S, Dunham GM, Chew FS: Traumatic lipohemobursa of the infrapatellar bursa. Radiol Case Rep. 2018, 13:513-5. 10.1016/j.radcr.2017.12.004

5. Colletti P, Greenberg H, Terk MR: MR findings in patients with acute tibial plateau fractures . Comput Med Imaging Graph. 1996, 20:389-94. 10.1016/s0895-6111(96)00054-7

6. Lee JH, Weissman BN, Nikpoor N, Aliabadi P, Sosman JL: Lipohemarthrosis of the knee: a review of recent experiences. Radiology. 1989, 173:189-91. 10.1148/radiology.173.1.2781006

7. Lombardi M, Cardenas AC: Hemarthrosis. StatPearls Publishing, Treasure Island, FL; 2021.

8. Tillinghast CM, Gary JL: Compartment syndrome of the lower extremity. Compartment Syndrome. Mauffrey C, Hak DJ, Martin III M (ed): Springer, Cham; 2019. 67-81. 10.1007/978-3-030-22331-1_8

9. Thawait SK, Vossen JA, Muro GJ, Karol I: MRI illustration of traumatic lipohemarthrosis of the wrist joint due to a scaphoid fracture. Radiol Case Rep. 2012, 7:688. 10.2484/rcr.v7i3.688

10. Frink M, Hildebrand F, Krettek C, Brand J, Hankemeier S: Compartment syndrome of the lower leg and foot. Clin Orthop Relat Res. 2010, 468:940-50. 10.1007/s11999-009-0891-X

11. Guo J, Yin Y, Jin L, Zhang R, Hou Z, Zhang Y: Acute compartment syndrome: cause, diagnosis, and new viewpoint. Medicine (Baltimore). 2019, 98:e16260. 10.1097/MD.0000000000016260

12. Al-Bashaireh AM, Haddad LG, Weaver M, Chengguo X, Kelly DL, Yoon S: The effect of tobacco smoking on bone mass: an overview of pathophysiologic mechanisms. J Osteoporos. 2018, 2018:1206235.

$10.1155 / 2018 / 1206235$ 